\title{
How do spinal schwannomas progress? The natural progression of spinal schwannomas on MRI
}

\author{
Kei Ando, MD, PhD, Shiro Imagama, MD, PhD, Zenya Ito, MD, PhD, \\ Kazuyoshi Kobayashi, MD, PhD, Hideki Yagi, MD, Tetsuro Hida, MD, Kenyu Ito, MD, \\ Mikito Tsushima, MD, Yoshimoto Ishikawa, MD, PhD, and Naoki Ishiguro, MD, PhD \\ Department of Orthopaedic Surgery, Nagoya University Graduate School of Medicine, Aichi, Japan
}

\begin{abstract}
OBJECTIVE Little is known about the progression of spinal schwannomas. The aim of this study was to determine the natural progression of spinal schwannomas and establish the risk of tumor growth.

METHODS This study retrospectively analyzed data from 23 patients (12 men and 11 women, 40-89 years old) with schwannomas detected by MRI. The mean follow-up period was 5 years (range 2-10 years). The absolute and relative growth rates of the tumors were calculated.

RESULTS The average tumor size was $1495 \mathrm{~mm}^{3}$ at the initial visit and $2224 \mathrm{~mm}^{3}$ at the final follow-up. The average absolute growth rate was $139 \mathrm{~mm}^{3}$ per year, and the average relative growth rate was $5.3 \%$ per year. Tumors were classified into 3 groups based on enhancement patterns: isointense/hyperintense (iso/high; 11 cases), rim enhancement when enhancement was peripheral (high/rim; 5 cases), and heterogeneous/heterogeneous (hetero/hetero; 7 cases) based on Gd-enhanced T2-weighted MRI. The average absolute growth rates of the 3 lesion groups were $588 \mathrm{~mm}^{3}, 957$ $\mathrm{mm}^{3}$, and $3379 \mathrm{~mm}^{3}$, respectively $(\mathrm{p}<0.01)$.

CONCLUSIONS Although the tumors classified as iso/high and high/rim on T2-weighted Gd-enhanced MR images were small and grew very little, most tumors with hetero/hetero classification increased in size. Hetero/hetero-type tumors should be followed closely and may require surgery.

http://thejns.org/doi/abs/10.3171/2015.3.SPINE141218
\end{abstract}

KEY WORDS intradural extramedullary tumors; spinal schwannomas; natural progression; oncology

$\mathrm{S}$ PINAL schwannomas (SCHs), which are considered benign tumors (WHO Grade I), are the most common intradural extramedullary spinal tumor, accounting for $55 \%$ of spinal tumors. ${ }^{3}$ Because most spinal SCHs are found incidentally in patients who show signs of radiculopathy or myelopathy, data on the natural growth of spinal SCHs are sparse, and it is not clear how these tumors progress. However, advances in neuroimaging using CT and MRI have increasingly identified patients with incidental spinal SCHs. Although surgery remains the first option for symptomatic spinal SCHs, the course of treatment for asymptomatic spinal SCHs is not clear. The aim of this study was to follow spinal SCHs using MRI to see how they progress in order to provide useful information for predicting the growth potential of spinal SCHs.

\section{Methods \\ Patient Population}

Between 2000 and 2012, we chose 23 patients whose MR or CT images led us to suspect they had spinal intradural extramedullary SCHs. The institutional review board of the University of Nagoya School of Medicine approved this study, and each patient provided informed consent prior to enrollment. We followed the patients for a minimum of 2 years without surgery. We excluded patients with neurofibromatosis Type 1 ( 3 patients) and Type 2 (2 patients), schwannomatosis (2 patients), and radiologically suspected meningioma (1 patient).

We followed all patients using serial MRI with sequences obtained in the sagittal, coronal, and axial planes

ABBREVIATIONS JOA = Japanese Orthopaedic Association; $\mathrm{SCH}=$ schwannoma; $\mathrm{T} 1 \mathrm{~W}=\mathrm{T} 1$-weighted; $\mathrm{T} 2 \mathrm{~W}=\mathrm{T}$-weighted .

SUBMITTED December 2, 2014. ACCEPTED March 26, 2015.

INCLUDE WHEN CITING Published online October 2, 2015; DOI: 10.3171/2015.3.SPINE141218. 
and using T1-weighted (T1W) (TR 400-700 msec; TE 9-25 msec), fast spin-echo T2-weighted (T2W) (TR 3000-5000 msec; TE 96-150 msec), and postcontrast (0.1-mmol/kg Gd-DTPA) T1W sequences. Two boardcertified neurosurgeons and 2 radiologists reviewed all images. These neurosurgeons had worked mainly as spine surgeons and had interpreted spine MR images daily in their clinical and research practices. The 2 reviewers (T.H. and M.T.) identified and characterized any abnormalities by consensus. Neither neurosurgeon had received information pertaining to the patient's age, sex, clinical history, or symptoms at the time of the interpretation. They identified each tumor twice in the 23 patients and assessed its location, volume, signal-intensity characteristics, and enhancement. We recorded tumor sizes as the average of the 2 reviewers' measurements, which were determined from the contrast-enhanced MR images.

For simplicity of data presentation, tumor sizes are presented as tumor volumes $(\mathrm{V})$, with $\mathrm{V}=(\mathrm{L} \times \mathrm{W} \times \mathrm{H})$ $\times 4 \pi / 3$, as calculated by the National Institutes of Health's image program (Scion ImageJ). We determined tumor growth rates using the following calculations:

Absolute growth rate $\left(\mathrm{mm}^{3} / \mathrm{yr}\right)=\frac{\mathrm{dv}(\text { final-initial })}{\mathrm{t}}$

Relative growth rate $(\% / \mathrm{yr})=\left(\sqrt[t]{\frac{V \text { final }}{\text { Vinitial }}}-1\right) \times 100$

where Vfinal is the tumor volume measured on the last follow-up visit, Vinitial is the tumor volume measured on the initial radiological study, and $t$ is the time interval in years between the initial and final imaging studies. ${ }^{5}$

The contrast signal characteristics were classified relative to the spinal cord: precontrast T1W images were classified as isointense (iso), hyperintense (high), or hypointense (low); and precontrast T2W images were classified as isointense (iso), homogeneous hyperintense (high), or heterogeneous intense (hetero). The contrast-enhancement patterns on postcontrast T1W images were classified as homogeneous (homo), as rim enhancement when enhancement was peripheral (rim), or as heterogeneous (hetero).
We classified spinal tumors based on their morphological characteristics and as intramedullary or extramedullary based on their location with respect to the spinal cord. We classified SCHs into 3 subgroups (iso/homo, high/rim, and hetero/hetero) based on their T2W/postcontrast T1W images $^{1}$ (Fig. 1).

We used the Japanese Orthopaedic Association (JOA) scoring system to evaluate the grade of neurological impairment. ${ }^{2}$ The scoring system has a maximum of 29 points for lumbar evaluation and 17 points for cervical and thoracic evaluations. The scoring system consists of subjective symptoms, clinical signs, restriction of ordinary daily life, and urinary bladder function. To present patients' neurological statuses, we converted their JOA scores to percentages by dividing their lumbar scores by 29 and their cervical and thoracic scores by 17 .

\section{Statistical Analysis}

Data were analyzed using the Statistical Program for the Social Sciences (SPSS) version 21 software package (SPSS Inc.). The mean values are presented as the mean \pm SD. We used the Mann-Whitney U-test and Student t-test to analyze differences between 2 groups, and the KruskalWallis test to analyze differences among 3 groups. The correlation between each parameter and absolute growth rate is analyzed through multiple linear regression analysis, which is determined by the value of correlation coefficient, i.e., weak $(0.2$ to $<0.4)$, moderate $(0.4$ to $<0.6)$, and strong $(0.6-1.0)$. The difference is considered significant when the $\mathrm{p}$ value is $<0.05$. The intraclass correlation coefficient 2-way mixed model on absolute agreement was used to analyze measurement reliability in tumor volume.

\section{Results}

\section{Clinical Manifestation}

Table 1 shows sex, mean age at final follow-up, duration of follow-up, tumor location, JOA score at final follow-up, tumor volume, absolute growth rate, and relative growth rate. There were 12 male and 11 female patients with an age range of 40-89 years (mean age, 68.6 years). The follow-up period averaged 61 months (range $24-120$ months). The majority of patients $(n=14,60.9 \%)$ had tumors in
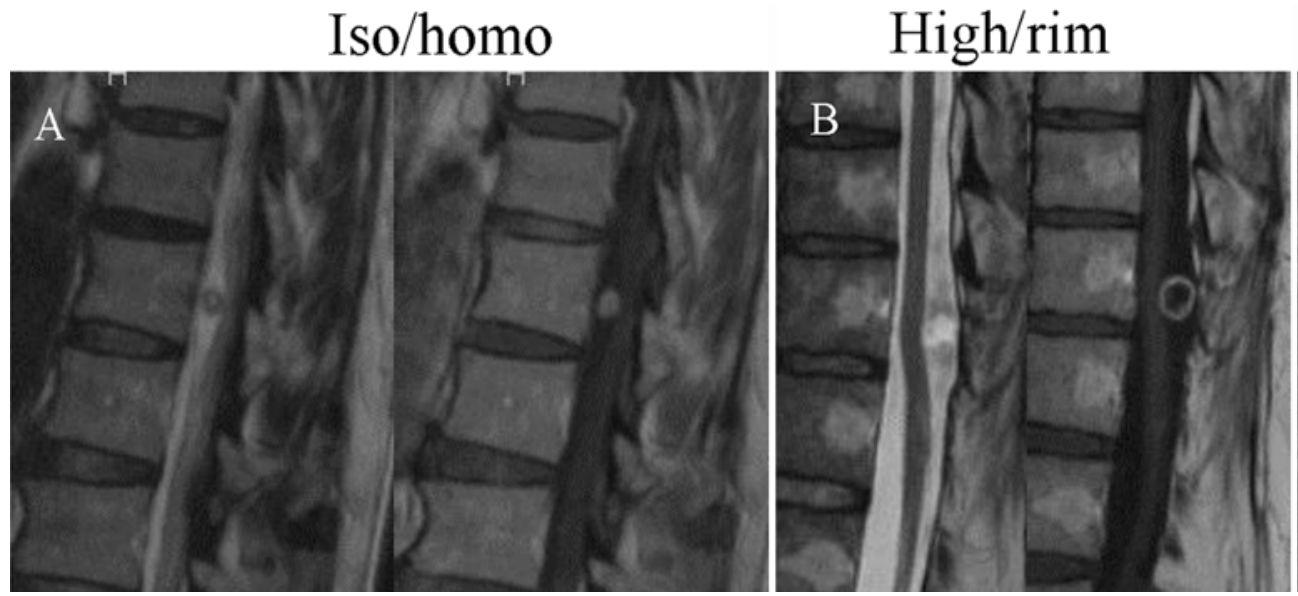

\section{Hetero/hetero}

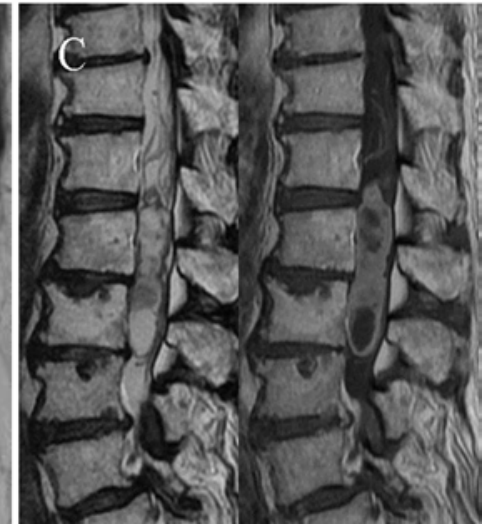

FIG. 1. T2W/postcontrast T1W images showing iso/homo (A), high/rim (B), and hetero/hetero (C) types of SCH. 
TABLE 1. Demographic and clinical data of patients with SCHs

\begin{tabular}{lc}
\hline \multicolumn{1}{c}{ Variable } & Value \\
\hline Sex & 12 \\
\hline $\mathrm{M}$ & 11 \\
\hline $\mathrm{F}$ & 68.6 \\
\hline Mean age at final FU, yrs \\
\hline FU, mos \\
\hline Mean, range \\
\hline Tumor location \\
\hline Cervical \\
\hline Thoracic \\
\hline Lumbar \\
\hline JOA score at final FU, \% \\
\hline Mean, range \\
\hline Tumor volume, mm ${ }^{3}$ \\
\hline Mean at initial examination, range \\
\hline Mean at final FU, range \\
\hline Absolute growth rate, mm ${ }^{3 / y r}$ \\
\hline Mean, range \\
\hline Relative growth rate, \%/yr \\
\hline Mean, range \\
\hline F
\end{tabular}

$\mathrm{FU}=$ follow-up.

the lumbar region, 6 patients $(26.1 \%)$ had tumors in the thoracic region (all lower thoracic), and 3 patients (13.0\%) had cervical tumors. The mean JOA score at final followup was $91.3 \%$ (range 31\%-100\%). Patients underwent MRI due to presenting complaints of neck pain (1 patient), low-back pain ( 9 patients), and leg pain or numbness (12 patients). One patient had severe bilateral lower-limb paresis but refused surgery. Only 1 patient with a tumor at L-5 ultimately underwent surgical excision of the tumor 3 years after the initial examination because of progressive leg pain. In this case, the pathology was schwannoma, and the mean tumor volume had significantly increased by the time of surgery $(\mathrm{p}<0.01)$. The intra- and interobserver reliabilities were 0.960 (Observer 1), 0.962 (Observer 2), and 0.966 (interobserver) for tumor volume.

\section{Findings on MRI}

On T1W images, all tumors were iso- or hypointense. Of the 23 tumors on T2W images, 11 (47.8\%) were isoin- tense, $5(21.7 \%)$ were homogeneously hyperintense, and $7(30.4 \%)$ were heterogeneously intense. Isointensity on T2W images was the most frequent signal intensity. On postcontrast T1W images, $11(47.8 \%)$ of the 23 tumors showed rim enhancement, 5 (21.7\%) showed homogeneous enhancement, and 7 (30.4\%) showed heterogeneous enhancement. As previously reported, ${ }^{1}$ MRI findings on $\mathrm{T} 2 \mathrm{~W}$ images of all tumors suspected to be SCHs corresponded with postcontrast T1W images.

\section{Relationship Between MRI Findings and Tumor Growth}

The SCHs that were homogeneously hyperintense (high) or heterogeneously intense (hetero) on T2W images were significantly larger than $\mathrm{SCH}$ that were isointense (iso) at the initial examination $(\mathrm{p}<0.01)$ (Table 2). The $\mathrm{SCH}$ that were isointense on $\mathrm{T} 2 \mathrm{~W}$ images increased very little in tumor volume (Fig. 2). However, the SCHs that were heterogeneously intense on T2W images had a significantly greater absolute growth rate, mainly due to the increase in volume of the cystic portion transformed from enhanced lesion (Fig. 3).

\section{Correlation Between Each Parameter and the Absolute Growth Rate}

The results of the association between each parameter and the absolute growth rate based on the multiple linear regression analysis are shown in Table 3 . The parameters of tumor volume (initial) and MRI findings were significantly positively correlated with the absolute growth rate $(p<0.05)$. The degree of each parameter's contribution to the absolute growth rate variation is as follows: tumor volume (initial) (correlation coefficient 0.703, strong), MRI findings (correlation coefficient 0.435 , moderate), age (correlation coefficient 0.073 , weak), and tumor location (correlation coefficient 0.000 , weak).

\section{Discussion}

Spinal SCHs are benign tumors that are typically intradural extramedullary, solitary, well-circumscribed, encapsulated, and located eccentrically on spinal nerve roots. ${ }^{4}$ Spinal SCHs and meningiomas account for $70 \%$ of intradural extramedullary tumors. ${ }^{8}$ The incidence of SCHs was reported to be approximately 4-5 times the incidence of meningiomas in Japan..$^{3,6}$ In the present study, we suspected that 23 of 24 radiologically observed, solitary, intradural extramedullary tumors were SCHs. The majority of

TABLE 2. MRI findings and tumor growth in patients with SCH

\begin{tabular}{lcccc}
\hline \multicolumn{1}{c}{ Variable } & Iso/Homo & High/Rim & Hetero/Hetero & p Value $^{*}$ \\
\hline No. of patients & 11 & 5 & 7 & NS \\
\hline Sex, M/F & $6: 5$ & $3: 2$ & $3: 4$ & NS \\
\hline Mean age, yrs & 70 & 63 & 70 & NS \\
\hline Tumor location, C/T/L & $1 / 4 / 6$ & $2 / 2 / 1$ & $0 / 0 / 7$ & $<0.01$ \\
\hline Initial tumor volume, $\mathrm{mm}^{3}$ & 588 & 957 & 3379 & $<0.01$ \\
\hline Absolute growth rate, $\mathrm{mm}^{3} / \mathrm{yr}$ & 25 & 124 & 334 & NS \\
\hline Relative growth rate, $\% / \mathrm{yr}$ & 4 & 7.2 & 6.7 & \\
\hline
\end{tabular}

$\mathrm{C}=$ cervical; $\mathrm{L}=$ lumbar; $\mathrm{NS}=$ not significant; $\mathrm{T}=$ thoracic.

${ }^{*}$ Kruskal-Wallis test. 


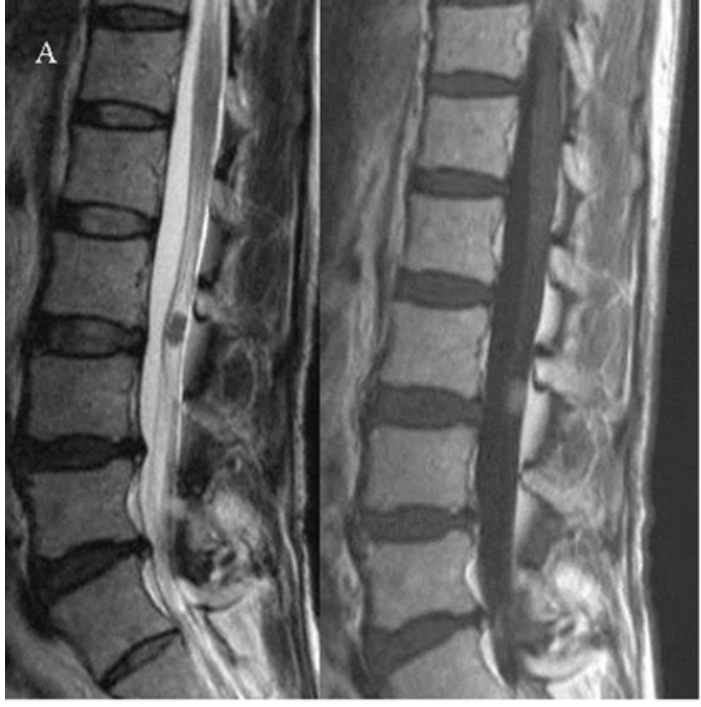

Initial

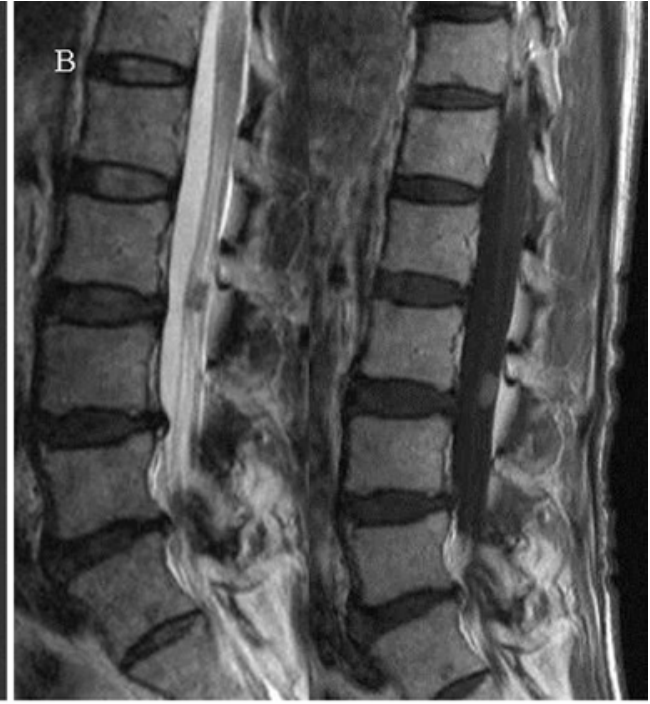

Final (9y)

FIG. 2. The tumor showed isointensity on $\mathrm{T} 2 \mathrm{~W}$ images and homogeneous enhancement on postcontrast $\mathrm{T} 1 \mathrm{~W}$ images, and it increased slightly in volume. $y=$ years.

the affected cord levels were in the lumbar region, similar to another report. ${ }^{3}$ Ozawa et al. reported a relative growth rate (percentage increase) of $2.3 \% \pm 5.5 \%$ for these tumors. ${ }^{7}$ Although all tumors in the current study had an average growth rate of $5.3 \%$, tumors with $\mathrm{T} 2 \mathrm{~W} /$ postcontrast T1W signal intensities of high/rim and hetero/hetero had $7.2 \%$ and $6.7 \%$ growth rates, respectively, and iso/homo tumors had only a $4.0 \%$ growth rate.

Spinal schwannomas that were homogeneously hyperintense (high) on T2W images had hypocellular lesions and cystic degeneration (the Antoni Type B tissue pattern). Those SCHs that were isointense on T2W images were highly cellular lesions (the Antoni Type A tissue pattern). Moreover, the Antoni Type A pattern, which was isoin- tense on T2W images, showed homogeneous enhancement on postcontrast T1W images. ${ }^{1}$ SCHs that were heterogeneously intense on $\mathrm{T} 2 \mathrm{~W}$ images increased in volume mainly due to the increase in volume of the cystic portion transformed from the enhanced lesion (Fig. 3). One study reported the Ki 67 labeling index as $<5 \%$ in most schwannomas. ${ }^{9}$ In the process of tumor growth, the tumor may necrose during cell division and develop cystic properties, contributing to the increase in volume. This is why the volume of tumors that were heterogeneously intense on their T2W images increased at follow-up. Tumors that were homogeneously hyperintense (high) on the T2W images may be transformed to those that are heterogeneously intense on T2W images. Tumors with isointensity on T2W images

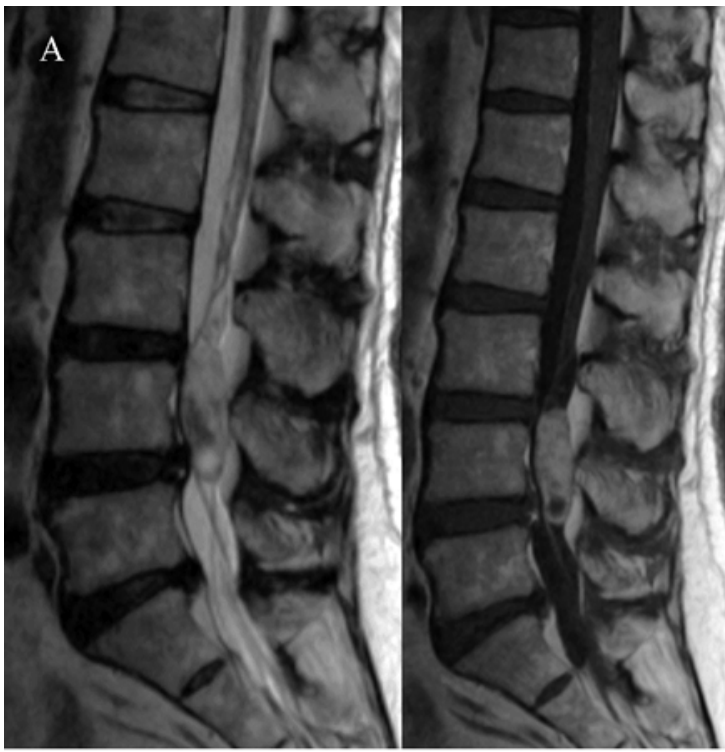

Initial

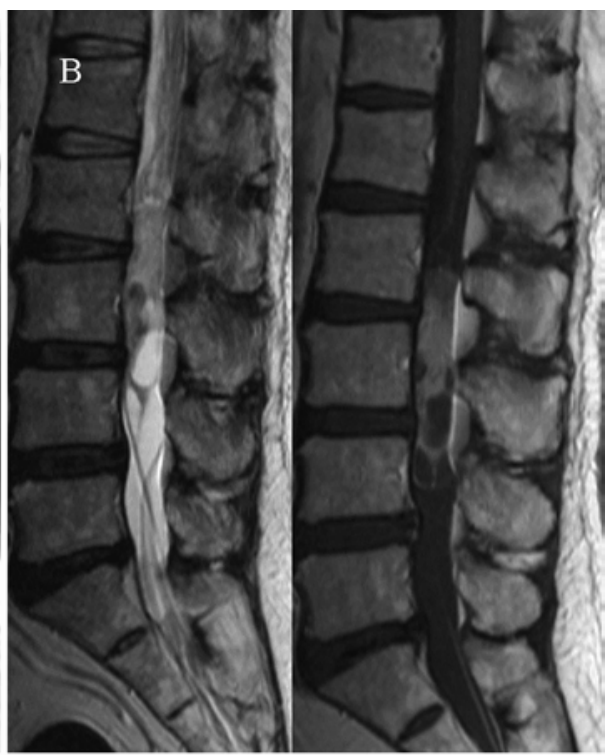

Final (8y)

FIG. 3. Tumors that were heterogeneously intense on T2W images and postcontrast T1W images had a significantly increased volume and absolute growth rate, mainly due to the increase in volume of the cystic portion transformed from the enhanced lesion. 
TABLE 3. Multiple linear regression analysis showing the correlation between the measured parameters and absolute growth rate

\begin{tabular}{llccc}
\hline Independent Variable & Dependent Variable & Estimate of Coefficient & t Statistic & $p$ Value \\
\hline Age & Absolute growth rate & 0.073 & 0.438 & 0.666 \\
\hline Tumor location & Absolute growth rate & 0 & 0 & 1 \\
\hline Tumor volume, initial & Absolute growth rate & 0.703 & 4.188 & $<0.01$ \\
\hline MRI findings & Absolute growth rate & 0.435 & 2.214 & 0.038 \\
\hline
\end{tabular}

may have enlarged further if we had followed them for a longer period, although we saw little increase in volume of those tumors during this study.

The limitations of this study were its small size and the fact that our data set did not include a pathological diagnosis because of conservative follow-up. Obviously, the pathological diagnosis would be ideally obtained by biopsy in nonsurgically observed cases, but biopsy is difficult in cases of intradural extramedullary spinal tumors. To our knowledge, this is the first report to examine the natural progression of suspected spinal $\mathrm{SCH}$ and their mechanisms of increase. We may ascertain more differences in these tumors by measuring cell reproductive rates (MIB-1 index) of the 3 groups on MR images; this provides a topic for future research.

We recommend closely following these tumors with imaging studies, especially large-volume tumors that are heterogeneously intense on T2W images, with the Antoni Type A pattern of highly cellular lesions and the Antoni Type B pattern of lesions with low cell density on pathological examination. The physician should consider excision if the tumor continues to grow with clinical symptoms or when the tumor causes significant compression of the spinal cord and cauda equina.

\section{Conclusions}

The majority of asymptomatic SCHs have only minimal growth and do not need surgical intervention. Physicians should monitor clinical symptoms and perform repeated imaging studies when patients have large-volume tumors that are heterogeneously intense on their T2W images, indicating an Antoni Type A pattern of high celldensity lesions and an Antoni Type B pattern of low celldensity lesions on pathological examination. Radiological features, such as heterogeneously intense on T2W images, may provide useful information to predict the growth potential of spinal SCHs. Surgical removal should be considered if continued tumor growth with symptoms occurs or when the tumor causes significant compression of the spinal cord and cauda equina.

\section{References}

1. Ando K, Imagama S, Ito Z, Hirano K, Tauchi R, Muramoto
A, et al: Differentiation of spinal schwannomas and myxopapillary ependymomas: MR imaging and pathologic features. J Spinal Disord Tech 27:105-110, 2014

2. Izumida S, Inoue S: [Assessment of treatment for low back pain.] J Jpn Orthop Assoc 60:391-394, 1986 (Jpn)

3. Hirano K, Imagama S, Sato K, Kato F, Yukawa Y, Yoshihara $\mathrm{H}$, et al: Primary spinal cord tumors: review of 678 surgically treated patients in Japan. A multicenter study. Eur Spine J 21:2019-2026, 2012

4. Jinnai T, Koyama T: Clinical characteristics of spinal nerve sheath tumors: analysis of 149 cases. Neurosurgery 56:510515,2005

5. Nakamura M, Roser F, Michel J, Jacobs C, Samii M: The natural history of incidental meningiomas. Neurosurgery 53:62-71, 2003

6. Ozawa H, Aizawa T, Kanno H, Sano H, Itoi E: Epidemiology of surgically treated primary spinal cord tumors in Miyagi, Japan. Neuroepidemiology 41:156-160, 2013

7. Ozawa H, Onoda Y, Aizawa T, Nakamura T, Koakutsu T, Itoi E: Natural history of intradural-extramedullary spinal cord tumors. Acta Neurol Belg 112:265-270, 2012

8. Simeone FA: Intraspinal neoplasms, in Rothman RH, Simeone FA (eds): The Spine. Philadelphia: Saunders, 1975

9. Torres-Mora J, Dry S, Li X, Binder S, Amin M, Folpe AL: Malignant melanotic schwannian tumor: a clinicopathologic, immunohistochemical, and gene expression profiling study of 40 cases, with a proposal for the reclassification of "melanotic schwannoma.” Am J Surg Pathol 38:94-105, 2014

\section{Disclosure}

The authors report no conflict of interest concerning the materials or methods used in this study or the findings specified in this paper.

\section{Author Contributions}

Conception and design: Ando. Acquisition of data: Ando, Tsushima, Ishikawa. Analysis and interpretation of data: Ando, Kobayashi. Critically revising the article: Hida. Reviewed submitted version of manuscript: Z Ito, Ishiguro. Statistical analysis: Yagi, K Ito. Study supervision: Imagama.

\section{Correspondence}

Shiro Imagama, Department of Orthopaedic Surgery, Nagoya University Graduate School of Medicine, 65 Tsurumai Showa-ward, Aichi 466-8550, Japan. email: imagama@med.nagoya-u.ac.jp. 